\title{
The role of collective effects and secular mass migration on galactic transformation
}

\author{
Xiaolei Zhang ${ }^{1}$ and Ronald J. Buta ${ }^{2}$ \\ ${ }^{1}$ Department of Physics and Astronomy, George Mason University, Fairfax, VA 22030, USA \\ email: xzhang5@gmu.edu \\ ${ }^{2}$ Department of Physics \& Astronomy, University of Alabama, \\ Box 870324, Tuscaloosa, AL, USA
}

\begin{abstract}
During the lifetime of a galaxy, secular radial mass redistribution is expected to gradually build up a bulge and transform the Hubble type from late to early. The dominant dynamical process responsible for this transformation is a collective instability mediated by density-wave collisionless shocks (Zhang 1996, 1998, 1999). The ability of this new mechanism to secularly redistribute the STELLAR mass provides a general pathway for the formation and evolution of the majority of Hubble types, ranging from late type disk galaxiess to disky ellipticals. ATLAS ${ }^{3 D}$ results (Cappellari et al. 2013) showed that spirals and S0s and disky ellipticals form a continuous trend of evolution which also coincides with the aging of the stellar population of galactic disks. The importance of stellar accretion is also revealed in the results of the COSMOS team which showed that the evolution of the black-hole-mass/bulge-mass correlation since $z=1$ was mainly due to the mass redistribution on pre-existing STELLAR disks which were already in place by $z=1$ (Cisternas et al. 2011). The weaker correlation between the masses of late-type bulges and AGNs observed at any given epoch in our view is a result of the quicker initial onset of accretion events in AGN disks compared to that in galactic disks, since the dynamical timescale is shorter for smaller AGN accretion disks.

The same secular dynamical process can produce and maintain the well-known scaling relations and universal rotation curves of observed galaxies during their Hubble-type transformation (Zhang 2008), as well as reproduce many other observed structural and kinematic properties of galaxies such as the size-line-width relation of the interstellar medium and the agevelocity dispersion relation of solar neighborhood stars in our own Galaxy. A by-product of this analysis is a powerful new method for locating the multiple corotation resonances in galaxies (Zhang \& Buta 2007; Buta \& Zhang 2009).

The current work also highlights the connection between collective effects in galactic dynamics and nonequilibrium phase transition processes in other branches of physics such as fluid turbulence and spontaneous breaking of gauge symmetry in high-energy physics. The continuous build-up of the Hubble sequence of galaxies through secular mass accretion also hints at the baryonic nature of galactic dark matter and poses challenges to the existing LCDM paradigm, since the well-known adiabatic compression process during baryonic mass inflow produced by secular evolution would lead to a concentration of the cold dark matter to the central region of early-type galaxies, which is not observed.
\end{abstract}

\section{References}

Buta, R. J. \& Zhang, X. 2009, ApJS 182, 559

Cappellari. M., et al. 2013, MNRAS 432, 1862

Cisternas, M., et al. 2011, ApJ 726, 57; ApJ 741, L11

Zhang, X. 1996, ApJ 457, 125; 1998, ApJ 499, 93 ; 1999, ApJ 518, 613

Zhang, X. 2008, PASP 120, 121

Zhang, X. \& Buta, R. J. 2007, AJ 133, 2584 